\title{
Angle-Resolved Reflectivity Analysis of Textured Substrates for Liquid-Phase Crystallized Silicon Thin-Film Solar Cells
}

\author{
David Eisenhauer, Grit Köppel, Bernd Rech, and Christiane Becker \\ Helmholtz-Zentrum Berlin für Materialien und Energie GmbH, Kekuléstr. 5, 12489 Berlin \\ david.eisenhauer@helmholtz-berlin.de
}

\begin{abstract}
Angular dependency of the reflectivity of two distinct nano-textured and planar reference liquid-phase crystallized silicon solar cells are investigated. We show that nano-textured layers outperform planar devices for all incident angles.
\end{abstract}

OCIS codes: $040.5350,310.6845$

\section{Introduction}

Liquid-phase crystallization (LPC) of silicon on glass is a technology that allows manufacturing high-quality crystalline silicon thin-films between $5-40 \mu \mathrm{m}$ in thickness, so far leading to solar cell efficiencies of up to $12.1 \%$ [1]. With decreasing cell thicknesses, light-management measures, specifically anti-reflection and light-trapping, become more and more important. An efficient way for anti-reflection in LPC silicon thin-film solar cells is nano-structuring the glass-silicon interface. However, the electronic material quality can be reduced by introducing nano-structures at this interface [2].

Two approaches were recently introduced that combine high electronic material quality LPC silicon with nanostructures, namely the sinusoidal structure [3] and the smooth anti-reflective three-dimensional (SMART) texture [4]. In this contribution, we investigate the angular dependence of the anti-reflective properties of these two structured LPC silicon absorbers compared to an optimized planar reference sample.

\section{Experimental}

Two different structured superstrates were used, a sinusoidal texture and the SMART texture. Both nano-structures have been shown to allow the production of high-quality liquid-phase crystallized silicon on textured substrates. The production process of the textured superstrates is depicted in Fig. 1.

(a)

(b)

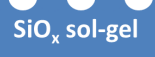

$\mathrm{SiO}_{\mathrm{x}} /$ glass

Nanoimprint lithography

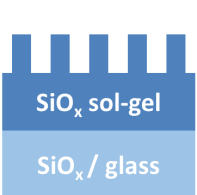

$\operatorname{SiN}_{x}$

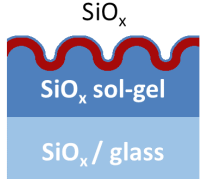

Spin-Coating

$\mathrm{TiO}_{x}$ sol-gel

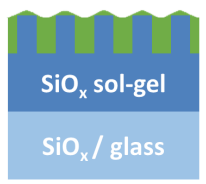

Sputtering

$\mathrm{SiO}_{x}$

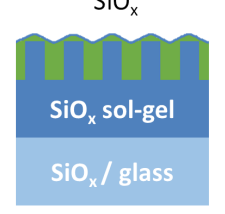

Fig. 1. Schematic production process of superstrates with (a) a sinusoidal texture and (b) comprising a SMART texture. 
For both textures, Corning Eagle XG glass sheets of $5 \times 5 \mathrm{~cm}^{2}$ are cleaned with the commercial cleaning agent Mucasol ${ }^{\circledR}$ and subsequently coated with a $250 \mathrm{~nm}$ thick $\mathrm{SiO}_{x}$ layer serving as diffusion barrier for glass impurities during cell processing. Subsequently, the nano-texture is imprinted into a high-temperature stable $\mathrm{SiO}_{x}$ sol-gel by UV nanoimprint lithography.

For the sinusoidal texture [Fig. 1(a)], the master structure was prepared by interference lithography [5]. Adapted from the optimized planar reference stack, the sinusoidal texture is coated with $70 \mathrm{~nm}$ of $\mathrm{SiN}_{x}$ and $10 \mathrm{~nm}$ of $\mathrm{SiO}_{x}$, serving as anti-reflective and passivation layers, respectively $[6,8]$.

For the SMART texture [(Fig. 1(b)], a nano-pillar array is used. This nano-pillar array surface is smoothed by spincoating a $\mathrm{TiO}_{x}$ precursor solution and subsequent thermal annealing. Thereby, the voids between the nano-pillars are preferentially filled and a smooth surface is formed. Finally, a $10 \mathrm{~nm}$ thick $\mathrm{SiO}_{x}$ passivation layer is sputtered onto the superstrate. For comparison, both textures exhibit a period of $750 \mathrm{~nm}$. For the SMART texture, the anti-reflective effect arises from the inter-mixed $\mathrm{SiO}_{x} / \mathrm{TiO}_{x}$ layer. Therefore, no additional $\mathrm{SiN}_{x}$ layer is needed.

For planar reference samples, a state-of-the-art interlayer stack of $250 \mathrm{~nm} \mathrm{SiO}_{x}, 70 \mathrm{~nm} \mathrm{SiN}_{x}$ and $10 \mathrm{~nm} \mathrm{SiO}_{x}$ is employed $[6,8]$. Using electron-beam evaporation with a substrate temperature of $T=600^{\circ} \mathrm{C}$, a $8 \mu \mathrm{m}$ thick silicon layer is deposited onto each superstrate. Liquid-phase crystallization is performed using a line-shaped laser beam of $808 \mathrm{~nm}$ wavelength and a scanning speed of $3 \mathrm{~mm} / \mathrm{s}$. Optical characterization was performed using a Perkin Elmer 1050 photospectrometer with an integrating sphere. For angle-resolved measurements, samples were placed on a rotatable sample holder in the center of the integrating sphere. The incident angle on the sample is offset by $8^{\circ}$, such that the specular reflectance cannot leave the integrating sphere. Transmitted light is absorbed using a black sheet behind the sample, allowing to directly measure reflectance.

The reflectivity $R(\theta)$ at the air-glass interface under illumination at an incident angle of $\theta$ is calculated using Fresnel's equations for $n_{1}=1.0$,

$$
\begin{aligned}
& R_{S}(\theta)=\left|\frac{\cos \theta-n_{2} \sqrt{1-\left(\frac{1}{n_{2}} \sin \theta\right)^{2}}}{\cos \theta+n_{2} \sqrt{1-\left(\frac{1}{n_{2}} \sin \theta\right)^{2}}}\right|^{2} \\
& R_{p}(\theta)=\left|\frac{\sqrt{1-\left(\frac{1}{n_{2}} \sin \theta\right)^{2}}-n_{2} \cos \theta}{\sqrt{1-\left(\frac{1}{n_{2}} \sin \theta\right)^{2}}+n_{2} \cos \theta}\right|^{2} \\
& R(\theta)=\frac{1}{2}\left(R_{S}(\theta)+R_{p}(\theta)\right) .
\end{aligned}
$$

Here, $R_{s}(\theta), R_{p}(\theta)$, and $n_{2}$ are the reflectivity for $s$ - and $p$-polarized light, and the refractive index of the glass superstrate, respectively.

\section{Results}

Figure 2 shows the angular dependency of the mean reflectivity between $400 \mathrm{~nm}$ and $600 \mathrm{~nm}$ of silicon absorbers on a sinusoidal textured (blue diamonds), SMART textured (green squares) and planar (black circles) superstrates as a function of incident angle. The reflection of light reflected at the air-glass interface, is plotted in Fig. 2 for reference (black line).

It is seen that for every angle of incidence, the textured samples efficiently reduce reflection compared to the optimized planar interlayer stack. Mean reflectivities lower than $10 \%$ are observed up to incident angles up to $50^{\circ}$ for the sinusoidal texture and up to $60^{\circ}$ for the SMART texture, despite the SMART texture having smooth interfaces. Of these, $3-4 \%$ of the incident light is already reflected at the air-glass interface depending on the angle of incidence. This loss mechanism could be addressed by an additional anti-reflective layer at this interface, e.g. a moth-eye texture [7].

For very high incident angles, the difference between textured and planar samples is reduced. In this range, the total reflection is dominated by reflection at the air-glass interface. 


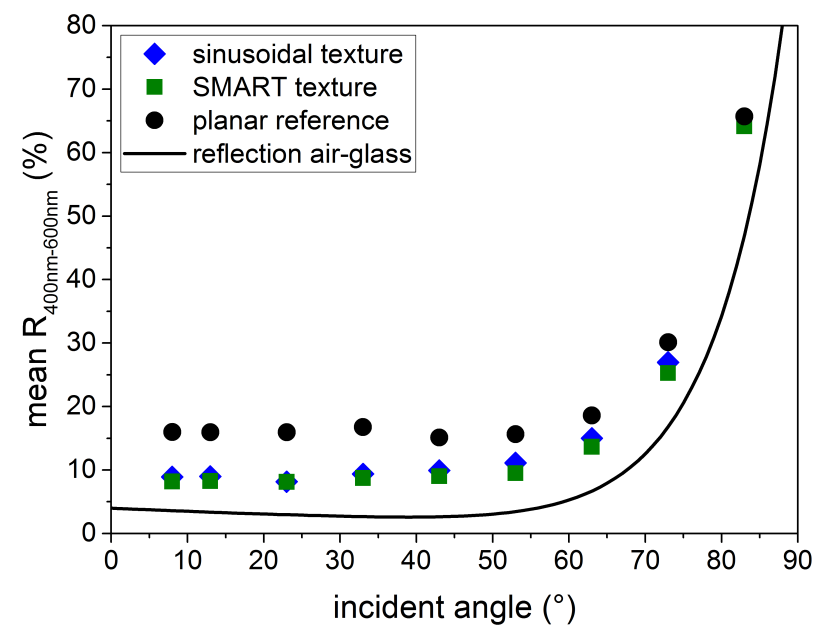

Fig. 2. Angular dependency of the mean reflectivity between $400 \mathrm{~nm}-600 \mathrm{~nm}$ for the sinusoidal texture (blue diamonds), the SMART texture (green squares) and the planar reference samples (black dots). For clarity, the reflection at the air-glass interface is plotted (black line).

\section{Conclusion}

Anti-reflective measures at the glass-silicon interface in liquid-phase crystallized silicon thin-film solar cells were evaluated by means of angle-resolved reflectivity measurements. We demonstrated that nano-structured cells efficiently reduce reflection, with total reflection of less than $10 \%$ remaining stable for incident angles up to $50^{\circ}$, compared to about $15 \%$ in optimized planar reference cells.

\section{References}

1. T. Frijnts, S. Kühnapfel, S. Ring, O. Gabriel, S. Calnan, J. Haschke, B. Stannowski, B. Rech, and R. Schlatmann, "Analysis of photo-current potentials and losses in thin film crystalline silicon solar cells," Sol. Energy Mat. Sol. Cells 143, 457-466 (2015).

2. V. Preidel, D. Amkreutz, J. Haschke, M. Wollgarten, B. Rech, and C. Becker, "Balance of optical, structural, and electrical properties of textured liquid phase crystallized Si solar cells," J. Appl. Phys. 117, 225306 (2015).

3. G. Köppel, B. Rech, and C. Becker, "Sinusoidal nanotextures for light management in silicon thin-film solar cells," Nanoscale 8, 8722-8728 (2016).

4. D. Eisenhauer, G. Köppel, K. Jäger, D. Chen, O. Shargaieva, B. Rech, and C. Becker, "Smooth anti-reflective three-dimensional textures for liquid-phase crystallized silicon thin-film solar cells on glass," manuscript submitted for publication.

5. A. Mellor, H. Hauser, C. Wellens, J. Benick, J. Eisenlohr, M. Peters, A. Guttowski, I. Tobías, A. Martí, A. Luque and B. Bläsi, Opt. Express 21, A295-A304, (2013).

6. J. Dore, D. Ong, S. Varlamov, R. Egan and M. A. Green, "Progress in Laser-Crystallized Thin-Film Polycrystalline Silicon Solar Cells: Intermediate Layers, Light Trapping, and Metallization," IEEE J. Photovoltaics 4, 3339 (2014).

7. S. J. Wilson and M. C. Hutley, "The Optical Properties of 'Moth Eye' Antireflection Surfaces," Optica Acta 29, 9931009 (1982).

8. D. Amkreutz, W. D. Barker, S. Kühnapfel, P. Sonntag, O. Gabriel, S. Gall, U. Bloeck, J. Schmidt, J. Haschke, and B. Rech, "Liquid-Phase Crystallized Silicon Solar Cells on Glass: Increasing the Open-Circuit Voltage by Optimized Interlayers for n- and p-Type Absorbers," IEEE J. Photovoltaics 5, 1757-1761 (2015). 